\title{
DIAMOND DETECTOR TECHNOLOGY: STATUS AND PERSPECTIVES ${ }^{*}$
}

\author{
D. Hits ${ }^{24^{* *}}$, A. Alexopoulos ${ }^{3}$ M. Artuso ${ }^{20}$, F. Bachmair ${ }^{24}$ L. Bäni'24, M. Bartosik3, \\ J. Beacham ${ }^{13}$, H. Beck²3, V. Bellini' ${ }^{2}$, V. Belyaev'2, B. Bentele19, P. Bergonzo'11, A. Bes ${ }^{27}$, \\ J-M. Brom7, M. Bruzzi ${ }^{4}$, G. Chiodini' ${ }^{26}$, D. Chren ${ }^{18}$, V. Cindro', G. Claus7, J. Collot ${ }^{27}$, \\ J. Cumalat ${ }^{19}$, A. Dabrowski'3, R. D'Alessandro4, D. Dauvergne' ${ }^{27}$, W. de Boer'10, S. Dick ${ }^{13}$, \\ C. Dorfer ${ }^{24}$, M. Dunser3, V. Eremin ${ }^{6}$, G. Forcolin ${ }^{22}$, J. Forneris ${ }^{15}$, L. Gallin-Martel27, \\ M-L. Gallin-Martel27, K.K. Gan13, M. Gastal3, C. Giroletti'7, M. Goffe7, J. Goldstein'17, \\ A. Golubev , A. Gorišek9, E. Grigoriev8, J. Grosse-Knetter²3, A. Grummer ${ }^{21}$, M. Guthoff 3 , \\ I. Haughton'22, B. Hiti9, M. Hoeferkamp21, T. Hofmann'3, J. Hosslet7, J-Y. Hostachy27, \\ F. Hügging1, C. Hutton'17, J. Janssen', H. Kagan ${ }^{13}$, K. Kanxheri' ${ }^{28}$, G. Kasieczka ${ }^{24}$, R. Kass'13, \\ M. Kis5, G. Kramberger9, S. Kuleshov8, A. Lacoste' ${ }^{27}$, S. Lagomarsino4, A. Lo Giudice'5,

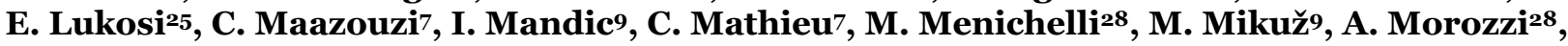

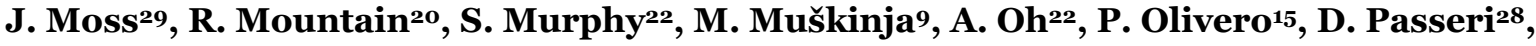

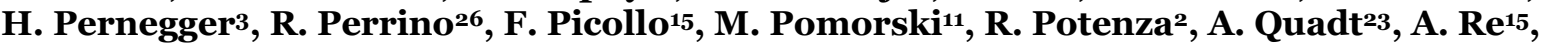 \\ M. Reichmann'24, G. Riley²5, S. Roe3, D. Sanz²4, M. Scaringella4, D. Schaefer3, C.J. Schmidt5, \\ S. Schnetzer14, S. Sciortino4, A. Scorzoni ${ }^{28}$, S. Seidel21, D.S. Smith'13, L. Servoli'², B. Sopko'18,

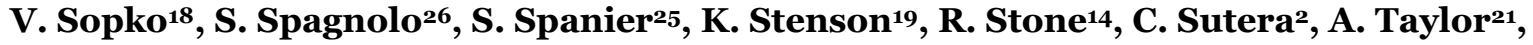 \\ B. Tannenwald13, M. Traeger5, D. Tromson'11, W. Trischuk ${ }^{16}$, C. Tuve' ${ }^{2}$, J. Velthuis' ${ }^{17}$,

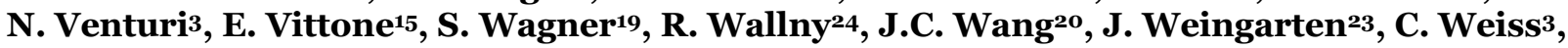 \\ N. Wermes' ${ }^{1}$ M. Yamouni' ${ }^{27}$, M. Zavrtanik9
}

${ }^{1}$ Universität Bonn, Bonn, Germany, ${ }^{2}$ INFN/University of Catania, Catania, Italy, ${ }^{3}$ CERN, Geneva, Switzerland, 4INFN/University of Florence, Florence, Italy, 5GSI, Darmstadt, Germany, ${ }^{6}$ Ioffe Institute, St. Petersburg, Russia, 7IPHC, Strasbourg, France, ${ }^{8}$ ITEP, Moscow, Russia, 9Jožef Stefan Institute, Ljubljana, Slovenia,

${ }^{10}$ Universität Karlsruhe, Karlsruhe, Germany, ${ }^{11}$ CEA-LIST Technologies Avancees, Saclay, France, ${ }^{12}$ MEPHI Institute, Moscow, Russia, 13The Ohio State University, Columbus, OH, USA,

${ }^{14}$ Rutgers University, Piscataway, NJ, USA, ${ }^{15}$ University of Torino, Torino, Italy,

${ }^{16}$ University of Toronto, Toronto, ON, Canada, ${ }^{17}$ University of Bristol, Bristol, UK, ${ }^{18}$ Czech Technical Univ., Prague, Czech Republic, 19University of Colorado, Boulder, CO, USA, ${ }^{20}$ Syracuse University, Syracuse, NY, USA, ${ }^{21}$ University of New Mexico, Albuquerque, NM, USA, ${ }^{22}$ University of Manchester, Manchester, UK, ${ }^{33}$ Universität Goettingen, Goettingen, Germany,

${ }^{24 E T H ~ Z u ̈ r i c h, ~ Z u ̈ r i c h, ~ S w i t z e r l a n d, ~}{ }^{25 U n i v e r s i t y ~ o f ~ T e n n e s s e e, ~ K n o x v i l l e, ~ T N, ~ U S A, ~}{ }^{26}$ INFN-Lecce, Lecce, Italy, ${ }^{27}$ LPSC-Grenoble, Grenoble, France, ${ }^{28}$ INFN-Perugia, Perugia, Italy, ${ }^{29}$ California State University, Sacramento, USA

\begin{abstract}
The radiation tolerance of chemical vapor deposition (CVD) diamond against different particle species and energies has been studied in beam tests and is presented. We also present beam test results on signal size as a function of incident particle rate in charged particle detectors based on un-irradiated and irradiated poly-crystalline CVD diamond over a range of particle fluxes from $2 \mathrm{kHz} / \mathrm{cm}^{2}$ to $20 \mathrm{MHz} / \mathrm{cm}^{2}$. The pulse height of the sensors was measured using readout electronics with a peaking time of $6 \mathrm{~ns}$. In addition, the functionality of poly-crystalline CVD diamond ${ }_{3} D$ devices is demonstrated in beam tests and ${ }_{3} D$ diamond detectors are shown to be a promising technology for applications in future high rate/high intensity experiments.
\end{abstract}

Key words: Poly-crystalline CVD diamond, rate dependence, ${ }_{3} D$ sensors, radiation tolerance

\section{INTRODUCTION}

Diamond is a material with extraordinary properties which rival any other semiconductor material. The low dielectric constant, high heat conductivity, large band gap, high threshold displacement energy, high electron and hole mobility make it an especially attractive candidate for particle detection in the high radiation environments.

Since its formation in 1994 [1], the RD42 collaboration at CERN has investigated Chemical Vapor Deposition (CVD) diamond for applications in collider experiments that include among others charged particle tracking, beam monitoring and luminosity measurement. Here we describe the highlights of these developments.

\footnotetext{
* This paper was presented at the Sixth International Conference on Radiation and Applications in Various Fields of Research (RAD 2018), Ohrid, Macedonia, 2018.

**dmitry.hits@phys.ethz.ch
} 


\section{RADIATION TOLERANCE}

The innermost regions of current and future hadron colliders have unprecedented radiation levels. The detectors at $3 \mathrm{~cm}$ radius from the beam axis at the High Luminosity Large Hadron Collider (HL-LHC) are expected to receive total fluence $2.3 \times 10^{16} \mathrm{n}_{\mathrm{eq}} / \mathrm{cm}^{2}$ during their lifetime [2]. The range of particle species contributing to the damage of the detectors is fairly broad, with the main contribution coming from charged hadrons while the other species (neutrons, electrons and photons) also have non-negligible effects. Therefore, tracking, beam monitoring, and luminosity detectors that occupy those regions should possess a sufficient degree of radiation tolerance and they should be tested against a broad spectrum of particles.

The RD42 collaboration has shown that photons do minimal damage to the diamond sensors at up to 10 MRad [1]. The collaboration has also published the diamond radiation damage factor for $24 \mathrm{GeV}$ protons [3]. Recently the collaboration has measured the radiation tolerance of polycrystalline and single crystal CVD diamonds against protons with energies $25 \mathrm{MeV}$, $70 \mathrm{MeV}$, and $800 \mathrm{MeV}$ protons, as well as against reactor neutrons $[4,5]$.

\subsection{Experimental details}

The ratio of the collected charge to the expected generated charge per unit length of the sensor (also known as Charge Collection Distance (CCD)) of nonirradiated samples was measured first, in order to determine the initial charge collection properties of the diamond. Prior to each irradiation the metal contacts were stripped, in order to reduce the "cool down" time. After each irradiation the samples were acid cleaned and re-metallized with a strip metallization pattern on the "growth" side and a backplane contact on the "substrate" side. The charge collection properties of the samples at $2 \mathrm{~V} / \mu \mathrm{m}$ bias field were then measured in a high energy $(100-200 \mathrm{GeV})$ hadron beam. The signals formed by charged carriers released through ionization by the beam particles were amplified by a multichannel VA2.2 amplifier [6]. The amplified signals were multiplexed, digitized with a Sirocco digitizer and saved to disk. Prior to each measurement the sensors were "pumped" in order to fill traps using a high intensity ${ }^{90} \mathrm{Sr}$ source for approximately 4 hours. The total dose received by the diamond sensor in the process was on the order of a few $\mu \mathrm{Gy}$. The pulse height was measured for both positive and negative bias polarity. A high resolution $(\sim 4 \mu \mathrm{m})$ beam telescope was used to predict the particle hit position in the device under test [7]. For more details on the experimental setup and procedures please refer to $[4,5]$.

\subsection{Analysis and results}

In order to determine the charge collection properties of the sensors, the raw data were preprocessed. The pedestal and common mode were subtracted, then, based on a predetermined cut, the hit clusters were formed in both the telescope planes and in the device under test. The hit clusters were used to align the telescope and the device under test. The events used for the alignment were discarded from the further analysis. The final pulse height distributions were formed by adding the amplitudes from the two strips with highest signals within 10 strips around the predicted hit position in the device under test.

Table 1 . The relative to the $24 \mathrm{GeV}$ proton irradiation hardness factors for different particle species [5]

\begin{tabular}{|l|r|}
\hline \multicolumn{1}{|c|}{ Particle species } & \multicolumn{1}{c|}{$\kappa$} \\
\hline $24 \mathrm{GeV}$ protons & 1 \\
$800 \mathrm{MeV}$ protons & $1.85 \pm 0.13$ \\
$70 \mathrm{MeV}$ protons & $2.5 \pm 0.4$ \\
$25 \mathrm{MeV}$ protons & $4.5 \pm 0.6$ \\
Reactor neutrons & $4.5 \pm 0.5$ \\
\hline
\end{tabular}

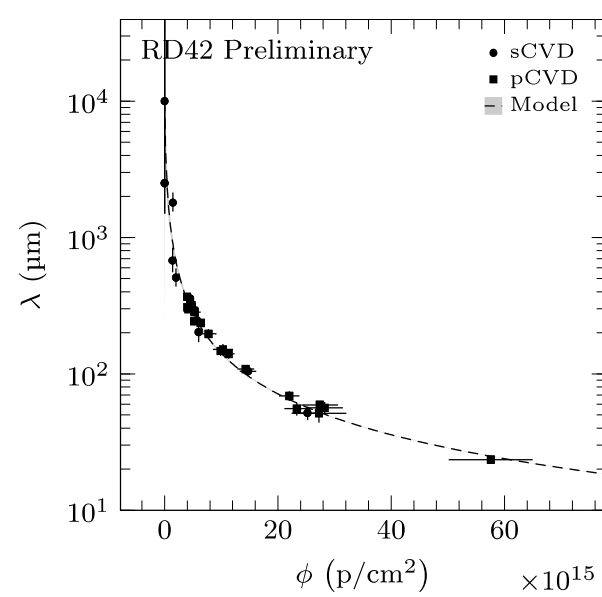

Figure 1. The average mean free path versus particle fluence of $24 \mathrm{GeV}$ protons. The fluences of reactor neutrons, 800 $\mathrm{MeV}$ protons and $70 \mathrm{MeV}$ protons, were scaled using the relative damage coefficient ( $\kappa$ ) in Table 1.

The average values of the distributions were converted to Schubweg or mean free path $(\lambda(\mu \mathrm{m}))$ by numerically inverting the following formula:

$$
Q_{\text {coll }}=\sum_{e, h} q n_{0} \lambda_{i}\left(1-\frac{\lambda_{i}}{d}\left(1-\exp \left(-\frac{d}{\lambda_{i}}\right)\right)\right)
$$

where $q(\mathrm{C})$ is the elementary charge, $n_{o}\left(\mu \mathrm{m}^{-1}\right)$ average number of electron hole pairs created by the detected charged particle per unit distance, $d(\mu \mathrm{m})$ is the sample thickness. The $Q_{\text {coll }}$ (average collected charged in Coulombs) is linearly related to the average pulse height through a calibration constant. For simplicity, the mean free path of electrons and holes were assumed equal.

In order to determine the damage constant $k$ $\left(\mathrm{cm}^{2} / \mu \mathrm{m}\right)$, the inverse mean free path values were plotted against the corresponding fluences $\Phi$ (particles $/ \mathrm{cm}^{2}$ ) and fitted with the following empirical equation $[8]$ : 


$$
\frac{1}{\lambda}=\frac{1}{\lambda_{0}}+k \Phi
$$

where $\lambda_{o}(\mu \mathrm{m})$ is the mean free path at zero fluence. The resulting damage factors for each particle species relative to the damage factor of $24 \mathrm{GeV}$ protons are shown in Table 1. By scaling the fluences of all the data with these relative damage factors, we can plot the data for all measured particle species on one graph (Figure 1).

\section{SigNAL PULSE HEIGHT VERSUS PARTICLE RATE}

The detectors in a collider experiment must operate in a wide range of particle rates, from few hundreds of $\mathrm{Hz} / \mathrm{cm}^{2}$ to several hundreds of $\mathrm{MHz} / \mathrm{cm}^{2}$. It is, therefore, necessary that the luminosity and the tracking detectors operate at a stable and high (>99\%) efficiency over this range of rates. The efficiency of the particle detectors depends on the pulse height distribution of the sensors and on the threshold of the readout electronics. Therefore, the dependence of the pulse distribution on incident particle rate should be studied over a wide range of rates prior to installing them in the collider experiment.

\subsection{Experimental details}

We studied the dependence of the pulse height on particle rate at the test beam facilities of Paul Scherrer Institute (PSI) in Villigen, Switzerland. The $\pi \mathrm{M} 1$ beam line of the High Intensity Proton Accelerator (HIPA) was tuned to provide positive pions $\left(\pi^{+}\right)$with a momentum of $260 \mathrm{MeV} / \mathrm{c}$ [9]. The rate of the $\pi^{+}$varied between $2 \mathrm{kHz} / \mathrm{cm}^{2}$ and $20 \mathrm{MHz} / \mathrm{cm}^{2}$ with the aid of two sets of collimators. The pulse height of the device under test was measured at several rate points.

Charged particle tracking was performed with compact beam telescope [4] having 4 tracking planes (2 upstream and 2 downstream from the device under test) each containing PSi46v2 [10] analog pixel readout chip mounted on a silicon sensor. Due to the small angle multiple scattering of the low momentum beam at PSI the hit resolution in the device under test was limited to $\sim 100 \mu \mathrm{m}$.

Two polycrystalline CVD diamond sensors were pre-selected for this test based on their leakage current and CCD characteristics. The samples were metalized on both sides with a single pad electrode of $3.5 \mathrm{x}$ $3.5 \mathrm{~mm}^{2}$ surrounded by a guard ring.

The samples were irradiated at Jožef Stefan Institute in Ljubljana, Slovenia. As with the proton irradiated samples (above) the contacts on the sensors were stripped, prior to irradiation, in order to accelerate the "cool down" process. After each irradiation the samples were re-metallized as described above.

The signals produced by $\pi^{+}$passing through the sample were amplified with a peaking time of $6 \mathrm{~ns}$ and a return to baseline of 20 ns. The signals were then digitized by a DRS4 evaluation board with a sampling rate of $2 \mathrm{GS} / \mathrm{s}$ [11].

\subsection{Results}

The pre-processing of the data is similar to the one described in Section 2.1 with a few nuances introduced by the high particle rates, differences in the trigger logic and the data-taking system. For details on data pre-processing and analysis please refer to reference [4]. For the final results, we used the mean of the pulse height distribution in a fiducial region that was slightly smaller than the metallized part of the diamond. Figure 2 shows the average pulse heights versus the particle rate for several rate points between few $\mathrm{kHz} / \mathrm{cm}^{2}$ and $20 \mathrm{MHz} / \mathrm{cm}^{2}$ for un-irradiated and irradiated polycrystalline CVD diamond with pad electrodes.

The result was obtained by varying the incident particle flux (both increasing and decreasing) several times, in order to ensure the repeatability. In order to emphasize the relative change, the pulse height for each point of a scan was arbitrarily normalized to the pulse height of the first lowest point rate for this scan.

For un-irradiated diamond, there is a slight $(\sim 2 \%)$ variation around the mean in the average pulse height. The variation of irradiated diamond the pulse height is $<1 \%$ over the whole range of incident fluxes. This measurement will be extended in the future to higher fluences.

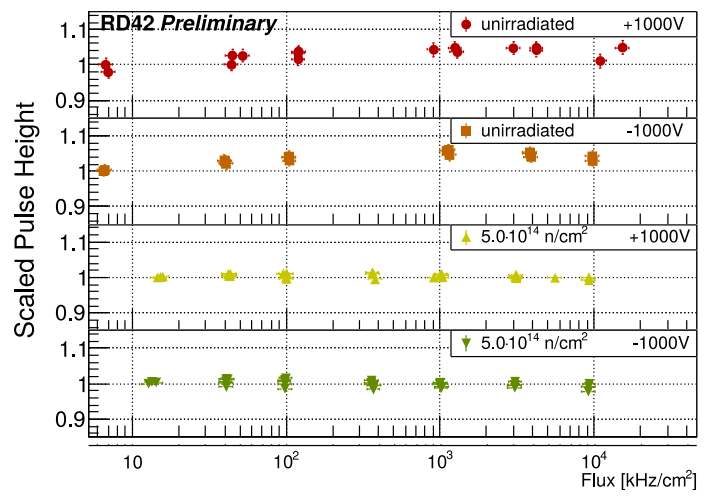

Figure 2. The average pulse height versus rate for an unirradiated and irradiated polycrystalline CVD diamond pad detector at positive and negative bias. The pulse height for each point of a scan was arbitrarily normalized to the pulse height of the first lowest point rate for this scan.

\section{3D DIAMOND PIXEL SENSORS}

$3 \mathrm{D}$ sensor design was proposed in 1997 [12], in order to overcome the limitation of decreasing mean free path in irradiated sensors. It is achieved by incorporating the bias and the readout electrodes into the sensor's bulk and, as a consequence, reducing the distance between the electrodes without reducing the thickness of the sensor. This results in a higher radiation tolerance.

Previously, the RD42 collaboration has demonstrated the successful implementation of a $3 \mathrm{D}$ structure in single crystal CVD diamond [13].

Here we show the results of the first implementation of the $3 \mathrm{D}$ structure in polycrystalline CVD diamond with a pixelated readout. 
4.1. Experimental details

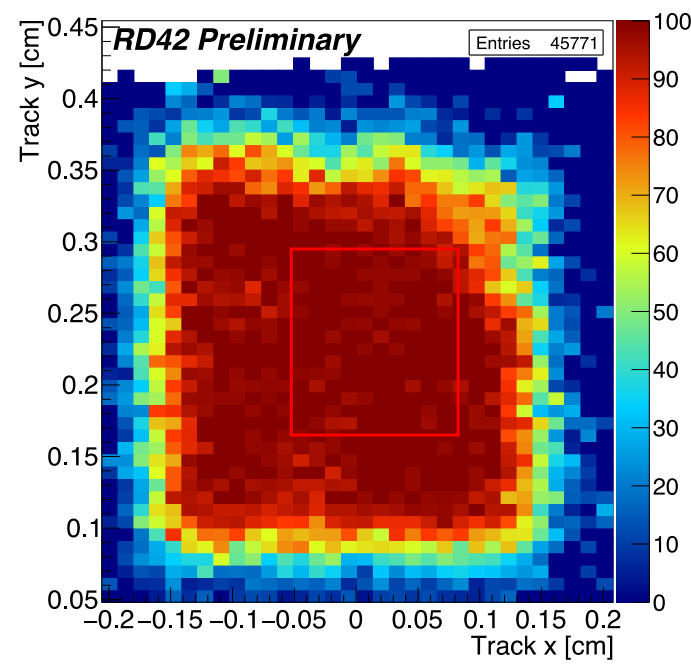

Figure 3. Detector efficiency versus predicted hit position at $67 \mathrm{kHz} / \mathrm{cm} 2$ particle rate. The red square identifies the fiducial region. The fiducial region avoids "hot" and "dead" pixels in the readout electronics. Detector bias $-55 \mathrm{~V}$.

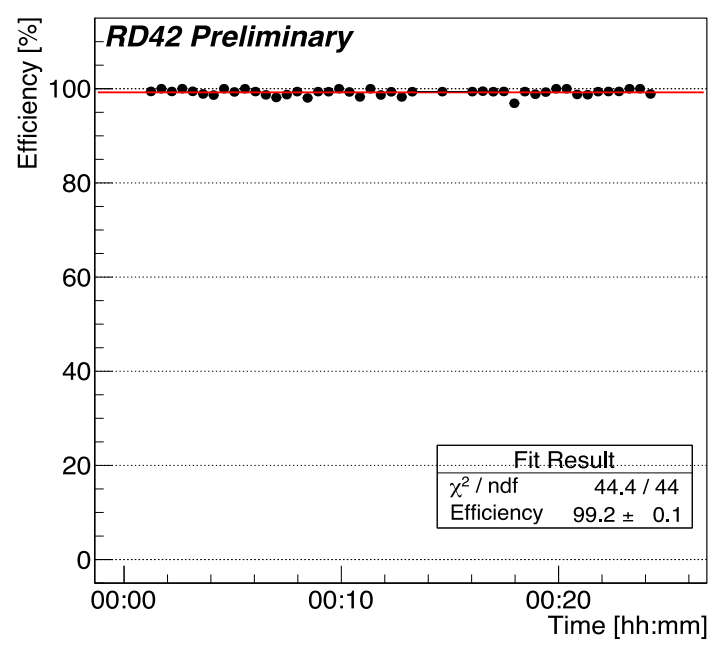

Figure 4. Detector efficiency in the fiducial region versus time at $67 \mathrm{kHz} / \mathrm{cm} 2$ particle rate. Detector bias $-55 \mathrm{~V}$.

A $5 \times 5 \times 0.5 \mathrm{~mm}^{3}$ polycrystalline CVD diamond sample manufactured by II-VI technologies [14] was chosen for the $3 \mathrm{D}$ detector based on charge collection properties. The $3 \mathrm{D}$ conductive electrodes with a resistivity of $0.1-1 \mathrm{ohm}-\mathrm{cm}$ in the diamond were fabricated with 100-fs laser pulses of $800-\mathrm{nm}$ wavelength. The laser light was focused to a spot $\leq 1 \mu \mathrm{m}$ diameter, while the sample was pulled throught it at 10 $\mu \mathrm{m} / \mathrm{s}$ [15]. The bias and readout electrodes were formed from opposite sides of the sample in such a way that only one end of the electrode reached either the bias or readout surface, the other end terminating 15 $\mu \mathrm{m}$ below the opposite surface. Four bias electrodes arranged in a square $50 \times 50 \mu \mathrm{m}^{2}$ pattern with one readout electrode in the middle formed one cell. All bias electrodes were connected together with a contact grid on the bias side. The readout electrodes were ganged in groups of six on the readout side. Such ganging was dictated by the readout chip geometry whose cells had pitches of $150 \mu \mathrm{m}$ and $100 \mu \mathrm{m}$. The total active area of the $3 \mathrm{D}$ pattern was $3.0 \times 3.1 \mathrm{~mm}^{2}$ or $60 \times 623 \mathrm{D}$ cells. PSI46dig chip [16] with a threshold tuned to 1500 electrons was used for readout. The sample was tested at $\pi \mathrm{M}_{1}$ beam line at PSI. The beam parameters and the tracking telescope were similar to those described in Section 3.1.

\subsection{Results}

The pre-processing of the data was similar to the one performed for the rate dependences studies described in section 3 . Only events with a single track in the telescope were selected for efficiency measurements. The detector under test was considered efficient only if there was a signal recorded within $\sim 1000 \mu \mathrm{m}$ of the predicted hit position. Figure 3 shows the efficiency map versus the predicted hit position. Most of the area of the $3 \mathrm{D}$ diamond is $>90 \%$ efficient. The area in the upper right corner had some known bump-bonding problems.

In order to determine the inherent efficiency of the 3D detector, we selected a fiducial region that avoids the areas with problematic bump-bonding, as well as the "hot" and the "dead" pixels in the readout chip. The resultant efficiency versus time, shown on Figure 4, is stable around $99.2 \%$ when the detector was operated at $-55 \mathrm{~V}$ bias. This represents a lower limit since the efficiency has not been corrected for cells with missing readout columns.

\section{CONCLUSION}

We have presented the most recent results of RD42 collaboration. The radiation tolerance of CVD diamond samples was measured for particle species. The pulse height of non-irradiated polycrystalline CVD diamonds varies less than $5 \%$ for particle rates from $10 \mathrm{kHz} / \mathrm{cm}^{2}$ to $10 \mathrm{MHz} / \mathrm{cm}^{2}$. The pulse height of the samples irradiated to neutron fluences from $5 \times 10^{13} \mathrm{n} / \mathrm{cm}^{2}$ to $4 \times 10^{15} \mathrm{n} / \mathrm{cm}^{2}$ varied less than $1 \%$ for the same range of pion fluxes. The $3 \mathrm{D}$ sensors based on polycrystalline CVD diamond had efficiency of $99.2 \%$ without removing the cells with missing readout columns.

Acknowledgements: RD42 collaboration gratefully acknowledges the staff at CERN for test beam time and their help in setting up the beam conditions. We would especially like to thank $H$. Wilkens, the test beam coordinator, for his assistance in making our tests a success. We also thank the beam line staff at the PSI High Intensity Proton Accelerator especially K. Deiters, M.Schwarz and D. Reggiani for their assistance in carrying out the diamond detector tests. We extend our gratitude to Dr. P. Salter and Prof. $M$. Booth of the Department of Engineering Science, University of Oxford for the laser processing of the $3 D$ columns in diamond and B. Harrop of Physics Department, Princeton University for bump bonding the ${ }_{3} D$ sensors to pixel readout chips. The research leading to these results received funding from the European Union's Horizon 2020 research and innovation program under grant agreement No. 654168. This work is also partially supported by ETH grant ETH-51 15-1, by Swiss Government Excellence 
Scholarship ESKAS No 2015-0808, the Royal Society grant UF120106, STFC grant ST/MOO3965/1/ and the U.S. Department of Energy through grant DESCoo10o61.

\section{REFERENCES}

1. M. H. Nazaré et al., Development of Diamond Tracking Detectors for High Luminosity Experiments at the LHC, R \& D Proposal, Geneva, Switzerland, 1994. Retrieved from: https://cds.cern.ch/record/293000/fil es/cer-0224986.pdf;

Retrieved on: 13.06 .2018

2. The Phase-2 Upgrade of the CMS Tracker, Technical Design Report, CERN, Geneva, Switzerland, 2018. Retrieved from: https://cds.cern.ch/record/2272264/fil es/CMS-TDR-014.pdf;

Retrieved on: Jun. 13, 2016

3. D. Meier et al., "Proton irradiation of CVD diamond detectors for high-luminosity experiments at the LHC," Nucl. Instrum. \& Meth., vol. A426, no. 1, pp. $173-180$, Apr. 1999.

DOI: 10.1016/So168-9002(98)01488-0

4. F. Bachmair, "CVD Diamond Sensors in Detectors for High Energy Physics," Ph.D. dissertation, ETH Zürich, 2016.

Retrieved from: http://inspirehep.net/record/1503510/ files/CERN-THESIS-2016-163.pdf; Retrieved on: Jun. 13, 2018

5. L. Bäni, "Top Quarks and Diamonds," Ph. D. dissertation, ETH Zürich, 2017.

Retrieved from: https://www.research-

collection.ethz.ch/bitstream/handle/20.500.11850/222 412/Top Quarks and Diamonds.pdf? sequence $=2$ \&isA llowed=y;

Retrieved on: Jun. 13, 2018

6. O. Toker et al, "VIKING, a CMOS low noise monolithic 128 channel frontend for Si-strip detector readout," Nucl. Instrum. \& Meth., vol. A340, no. 3, pp. 572 - 579, Mar. 1994.

DOI: 10.1016/0168-9002(94)90140-6

7. C. Colledani et al., "A submicron precision silicon telescope for beam test purposes," Nucl. Instrum. \& Meth., vol. A372, no. 3, pp. 379 - 384, Apr. 1996.
DOI: 10.1016/ 0168-9002(95)01414-4.

8. W. Adam, et al., "Pulse height distribution and radiation tolerance of CVD diamond detectors," Nucl. Instrum. \& Meth., vol. A447, no. 1-2, pp. 244-250, June 2000.

DOI: 10.1016/So168-9002(00)00195-9

9. $\pi M 1$ Beam Line, Paul Scherrer Institute, Villigen, Switzerland, 2018.

Retrieved from: https://www.psi.ch/sbl/pim1beamline; Retrieved on: Jun. 13, 2018

10. H. C. Kästli et al., "Design and performance of the CMS pixel detector readout chip," Nucl. Instrum. Meth., vol. A565, no. 1, pp. 188 - 194, Sep. 2006. DOI: $10.1016 /$ j.nima.2006.05.038

11. S. Ritt, DRS4 Evaluation Board, Paul Scherrer Institut, Villigen, Switzerland, 2018.

Retrieved from: http://www.psi.ch/drs/evaluationboard;

Retrieved on: Jun. 14, 2018

12. S. Parker, C. J. Kenney, J. Segal, " 3 -D: A proposed new architecture for solid state radiation detectors," Nucl. Instrum. Meth. Phys. Res., vol. A395, no. 3, pp. 328 - 343, Aug. 1997.

DOI: 10.1016/So168-9002(97)00694-3

13. F. Bachmair et al., "A 3D Diamond Detector for Particle Tracking," Nucl. Instrum. Meth. Phys. Res., vol. A786, pp. 97 - 104, Jun. 2015. DOI: 10.1016/j.nima.2015.03.033

14. II-VI Inc. official webpage, Saxonburg (PA), USA, 2018.

Retrieved from: https://www.ii-vi.com; Retrieved on: Jun. 14, 2018

15. M. J. Booth et al., "Study of cubic and hexagonal cell geometries of a $3 \mathrm{D}$ diamond detector with a proton micro-beam," Diam. Relat. Mater., vol. 77, pp. $137-145$, Aug. 2017. DOI: $10.1016 /$ j.diamond.2017.06.014

16. H. C. Kästli, "Frontend electronics development for the CMS pixel detector upgrade," Nucl. Instrum. Meth., vol. A731, pp. 88 - 91, Dec. 2013 DOI: 10.1016/j.nima.2013.05.056 